\title{
openheart Chronic heavy drinking and ischaemic heart disease: a systematic review and meta-analysis
}

\author{
Michael Roerecke, ${ }^{1,2}$ Jürgen Rehm ${ }^{1,2,3,4,5}$
}

To cite: Roerecke M, Rehm J. Chronic heavy drinking and ischaemic heart disease: a systematic review and metaanalysis. Open Heart 2014;1: e000135. doi:10.1136/ openhrt-2014-000135

- Additional material is available. To view please visit the journal online (http://dx. doi.org/10.1136/openhrt2014-000135).

Received 16 April 2014 Revised 14 July 2014 Accepted 16 July 2014

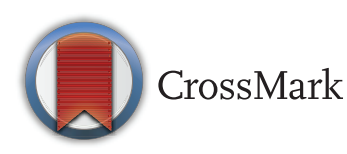

${ }^{1}$ Centre for Addiction and Mental Health (CAMH),

Toronto, Canada

${ }^{2}$ Dalla Lana School of Public Health (DLSPH), University of Toronto, Toronto, Canada

${ }^{3}$ Institute of Medical Science, University of Toronto,

Toronto, Canada

${ }^{4}$ Institute for Clinical Psychology and Psychotherapy, TU Dresden, Dresden, Germany ${ }^{5}$ Department of Psychiatry, University of Toronto, Toronto, Canada

Correspondence to Dr Michael Roerecke; m.roerecke@web.de

\section{ABSTRACT}

Previous meta-analyses have reported either a protective, neutral or detrimental association from chronic heavy drinking in relation to ischaemic heart disease (IHD). We investigated the potential for systematic error because of study design. Using MOOSE guidelines, studies were identified through MEDLINE, EMBASE and Web of Science up to end of March, 2014. Epidemiological studies reporting on chronic heavy drinking and IHD risk in population studies and samples of people with alcohol use disorder (AUD) were included. Random-effects metaanalysis was used to pool eligible studies. The $\mathrm{I}^{2}$ statistic was used to assess heterogeneity across studies. In total, 34 observational studies with 110570 chronic heavy drinkers and 3086 IHD events were identified. In population studies among men, the pooled risk for IHD incidence (fatal+non-fatal events) among chronic heavy drinkers (on average $\geq 60 \mathrm{~g}$ pure alcohol/day) in comparison to lifetime abstainers ( $n=11$ studies) was relative risk $(\mathrm{RR})=1.04(95 \% \mathrm{Cl} 0.83$ to $1.31,\left.\right|^{2}=54 \%$ ). Few studies were available for women. In patients with AUD, the risk of IHD mortality in comparison to the general population was elevated with a $\mathrm{RR}=1.62\left(95 \% \mathrm{Cl} 1.34\right.$ to $\left.1.95, \mathrm{I}^{2}=81 \%\right)$ in men and $R R=2.09\left(95 \% \mathrm{Cl} 1.28\right.$ to $\left.3.41, \mathrm{I}^{2}=67 \%\right)$ in women. There was a general lack of adjustment other than sex and age in studies among patients with AUD. There is no systematic evidence for a protective association from any type of chronic heavy drinking on IHD risk. Patients with AUD were at higher risk for IHD mortality, but better quality evidence is needed with regard to potential confounding.

\section{INTRODUCTION}

For some time the alcohol-heart relationship has been a controversial topic in heart disease epidemiology. ${ }^{1-3}$ The relationship between average alcohol consumption and ischaemic heart disease (IHD) is usually described as a J-shaped curve in epidemiological studies. Several meta-analyses have shown a protective association of some form of average alcohol consumption on IHD outcomes, ${ }^{2} 45$ with short-term experimental studies showing support for an effect on several surrogate biomarkers for elevated IHD risk ${ }^{6}$; these protective associations were quite strong and comparable to preventive measures for IHD, such as physical activity. ${ }^{7} 8$ The protective association seems to be not only relatively strong in magnitude, but also to include a wide range of average alcohol consumption. ${ }^{2} 9$ In particular, one meta-analysis ${ }^{4}$ concluded that there was an inverse relation with no detrimental effect on IHD from alcohol consumption even among chronic heavy drinkers $(\sim 25 \%$ risk reduction). However, another meta-analysis ${ }^{2}$ reached a very different conclusion with regard to IHD mortality among chronic heavy drinkers (no beneficial effect), and a similar conclusion with a risk reduction for IHD morbidity (beneficial effect). This underlines the importance of stratifying analyses by IHD outcome when examining evidence for the relationship between alcohol consumption and IHD. Additionally, using current abstainers as the referent (ie, the inclusion of former drinkers in the reference group) might lead to overestimation of any potential protective effects, ${ }^{10}$ and adjustment for potential confounding has not been optimal in many studies. ${ }^{13}$ The specific risk of chronic heavy drinking in comparison to abstainers taking into account these important conceptual and study design issues has not been systematically examined before and it is currently unclear whether chronic heavy drinking has a protective, neutral, or detrimental association with IHD. Furthermore, population studies often times miss many chronic heavy drinkers in order to maximise follow-up or because of other sampling issues. ${ }^{11} \mathrm{~A}$ good example of optimisation for follow-up availability is the Health Professionals Follow-Up Study. ${ }^{12}$ Inadvertently, these samples mostly contain more favourable drinking behaviour, such as low and regular alcohol consumption within a certain stratum of the socioeconomic continuum in high-income countries. However, as is increasingly evident in middle-income 
countries, this is not the drinking pattern observed globally, which is characterised by more heavy drinking occasions. ${ }^{13}$ Among participants missed in typical cohort studies is a subgroup of chronic heavy drinkers, namely people with alcohol use disorders (AUD), who may drink considerably more than the threshold for chronic heavy drinking we use in this meta-analysis $(\geq 60 \mathrm{~g}$ of pure alcohol per day). ${ }^{14-16}$ Several studies conducted among patients in treatment for AUD showed a relatively strong elevated IHD risk. ${ }^{15} 1718$

We hypothesised that there is no beneficial association with IHD risk in chronic heavy drinkers. Available evidence was systematically reviewed for IHD risk among chronic heavy drinkers in general population samples, and for people in AUD treatment (clinical samples). We stratified the analyses by reference group used for comparison and IHD outcomes (mortality vs morbidity).

\section{METHODS}

\section{Search strategy}

This meta-analysis followed the MOOSE guidelines. ${ }^{19}$ Updated search strategies from three previous meta-analyses ${ }^{2} 2021$ were used to identify observational studies reporting relative risk (RR) estimates for IHD in chronic heavy drinkers in comparison to abstainers in population samples, and to the general population in clinical samples up to 4th week of March 2014. Search terms included variations for the exposure (alcohol consumption), outcome (ischaemic heart disease) and study design (see online supplementary methods and figures S1 and S2 for details).

Inclusion criteria for the meta-analysis on chronic heavy drinking in population samples were: (1) prospective or historical cohort or case-control study design; (2) a measure of risk and its corresponding measure of variability was reported (or sufficient data to calculate these); (3) IHD was reported as a separate outcome (ie, excluding other cardiovascular diseases, such as stroke); (4) a risk estimate for chronic heavy drinking using any type of beverage ( $\geq 60 \mathrm{~g}$ pure alcohol per day on average based at least on a typical week's intake pattern) was reported among current drinkers; (5) a risk estimate for current or lifetime abstainers was reported; (6) estimates were stratified by sex and at least age-adjusted.

Inclusion criteria for the meta-analysis on AUD in clinical samples were: (7) prospective or historical cohort study; (8) a mortality risk estimate for patients with AUD was reported in comparison to the general population; (9) IHD was reported as a specific outcome; (10) a measure of risk and its corresponding measure of variability was reported (or sufficient data to calculate these); (11) estimates were stratified by sex and at least age-adjusted.

\section{Data extraction}

From all relevant articles we extracted authors' names, year of publication, country, calendar year(s) of baseline examination, follow-up period, setting, assessment of IHD, assessment of alcohol consumption or AUD diagnosis, mean and range of age at baseline, sex, number of observed IHD cases among participants by drinking group, number of total participants by drinking group, adjustment for potential confounders and RR and its SE. The most adjusted RR reported was used, and priority was given to estimates comparing chronic heavy drinking to lifetime abstainers.

\section{Definition of chronic heavy drinking and reference groups}

Heavy drinking is not uniformly defined. ${ }^{22}$ In this meta-analysis, chronic heavy drinking was defined as all drinking groups where the lower limit was at least $60 \mathrm{~g}$ / day. Clinical samples (patients with AUD) are generally missed in population studies, but they can be seen as similar in terms of heavy alcohol intake although a clear definition of alcohol intake in $\mathrm{g}$ /day is not possible. ${ }^{14}$ The clinical sample of patients with AUD was defined by a diagnosis of AUD by entering an alcohol treatment programme in a specialised treatment facility (this includes Diagnostic and Statistical Manual (DSM-III and IV) 'alcohol abuse and dependence' and International Classification of Diseases (ICD-9 and 10) 'alcohol use disorders'). Lifetime abstainers are defined as nondrinking groups where former drinkers were excluded. Current abstainers include both lifetime abstainers and former drinkers.

\section{Outcome ascertainment}

Self-reported IHD outcomes were excluded. In populations samples, IHD was defined based on standard criteria ascertained by death records (death certificate, and in some cases autopsy findings), standard criteria for myocardial infarction by WHO criteria, ${ }^{23}{ }^{24}$ ICD-7: 420422, ICD-8: 410-429, ICD-9: 410-414, ICD-10: I20-I25, or by committee decision based on medical records. In clinical samples, several versions of ICD were used in primary studies, but all studies were based on death certificates, sometimes using additional sources of information about the cause of death. For this meta-analysis we have categorised IHD outcomes into three groups: (1) IHD incidence (fatal or non-fatal events), (2) IHD mortality (fatal events only) and (3) IHD morbidity (nonfatal events only).

\section{Quality assessment}

Most quality scores are tailored for meta-analyses of randomised trials of interventions ${ }^{25-28}$ and many criteria do not apply to epidemiological studies like the ones examined here. Also, their use in meta-analyses remains controversial. ${ }^{28} 29$ Thus, quality assessment was incorporated differently by including quality components such as study design and alcohol measurement into the inclusion and exclusion criteria (see online supplementary table S1 and methods for details). One author performed the literature search and abstracted the data. To control for subjectivity, 10 papers were randomly 
selected and extracted by another author. No changes in abstraction were recorded. Information found in related papers from the same cohort was used where possible. Authors from primary studies were not contacted in case insufficient information was provided.

\section{Statistical analysis}

Standardised mortality ratios, HRs, ORs and RRs were treated as equivalent measures of risk. We calculated the overall pooled risk of IHD events associated with chronic heavy drinking stratified by sex and reference group, and conducted subanalyses stratified by assessment of IHD outcome (fatal and non-fatal events). IHD incidence included combined fatal and non-fatal events, or each respective outcome if only this was reported. When more than one estimate from primary studies was assigned to an IHD category, we combined the reported results using fixed-effects to derive one effect estimate per study separately for each analysis or subanalysis; chronic heavy drinking groups were combined using the method by Hamling et al. ${ }^{30}$ RRs were pooled across studies using inverse-variance weighted DerSimonian-Laird random-effect models to allow for between-study heterogeneity. ${ }^{31}$ Between-study heterogeneity was quantified using the $\mathrm{I}^{2}$ statistic. ${ }^{32} \mathrm{I}^{2}$ can be interpreted as the proportion of the total variation in the estimated effects for each study that is due to heterogeneity between studies. We conducted meta-regression analyses to identify study characteristics that influenced the association between chronic heavy drinking and IHD. Potential publication bias was examined using Egger's regression-based test. ${ }^{33}$ All regression-based tests were only conducted when 10 or more data points were available. Sensitivity analyses for the influence of single studies on the pooled RRs were conducted omitting studies one by one and re-estimating the pooled RR. All meta-analytical procedures were conducted on the natural log scale in Stata statistical software, V.11.2 (Stata Corp, College Station, Texas, USA).

\section{RESULTS}

\section{Literature search}

In total 34 unique articles meeting the inclusion criteria were used in this meta-analysis (see online supplementary table S1). Overall, 8 studies were conducted in the USA, 5 in Sweden and 4 in Japan, but articles from 20 countries were included altogether. Two papers provided pooled individual data from several studies. ${ }^{34} 35$ The analysis was based on 3086 observed IHD events (fatal and non-fatal) among 110570 chronic heavy drinkers. Average weighted follow-up time was 11.7 years in population samples and 10.4 in clinical samples. Regarding population samples, most excluded IHD disease at baseline or adjusted for prevalent IHD and smoking at baseline. Ten studies provided data from patients with AUD treatment (clinical samples), mostly standardised (by age and sex) mortality ratios (see online supplementary table S1 for details).

\section{Meta-analyses}

IHD risk among chronic heavy drinkers in men is displayed in table 1 . Among population samples, 11 studies provided data with lifetime abstainers as the reference group and 14 with current abstainers. The pooled risk for IHD incidence among chronic heavy drinkers in comparison to lifetime abstainers was $\mathrm{RR}=1.04,95 \%$ CI 0.83 to $1.31, \mathrm{n}=11$ studies, figure 1 ), IHD mortality risk was similar $(\mathrm{RR}=1.00,95 \% \quad \mathrm{CI} \quad 0.74$ to 1.36$)$. Heterogeneity was moderate (for IHD incidence $\left.\mathrm{I}^{2}=54 \%\right)$. In comparison to a reference group which contains current abstainers, pooled risks among chronic heavy drinkers were consistently lower (RR point estimates between 0.78 and 0.85 , IHD incidence was statistically significant with $\mathrm{RR}=0.83,95 \%$ CI 0.70 to 0.98 , figure 2) compared to examinations using lifetime abstainers as the reference group. Heterogeneity was more pronounced in studies using current abstainers as the reference group (for IHD incidence $\mathrm{I}^{2}=73 \%$ ). Five studies reported IHD morbidity risk among chronic

Table 1 Chronic heavy drinking and ischaemic heart disease (IHD) risk in men, 1967-2013

\begin{tabular}{|c|c|c|c|c|c|c|c|c|}
\hline $\begin{array}{l}\text { Heavy drinking } \\
\text { group, IHD end } \\
\text { point }\end{array}$ & Reference group & $\begin{array}{l}\text { Number of } \\
\text { studies }\end{array}$ & $\begin{array}{l}\text { Number of } \\
\text { events* }\end{array}$ & $\begin{array}{l}\text { Number of } \\
\text { chronic } \\
\text { heavy } \\
\text { drinkers }\end{array}$ & $\begin{array}{l}\text { Relative } \\
\text { risk }\end{array}$ & $95 \% \mathrm{Cl}$ & p Valuet & $I^{2}(\%)$ \\
\hline \multicolumn{9}{|c|}{ Chronic heavy drinking (population samples) } \\
\hline Incidence & Lifetime abstainers & 11 & 954 & 35756 & 1.04 & 0.83 to 1.31 & 0.016 & 54 \\
\hline Mortality & Lifetime abstainers & 5 & 618 & 34182 & 1.00 & 0.74 to 1.36 & 0.026 & 64 \\
\hline Morbidity & Lifetime abstainers & 3 & 299 & 471 & 1.13 & 0.78 to 1.63 & 0.97 & 0 \\
\hline Incidence & Current abstainers & 14 & 1268 & 50805 & 0.83 & 0.70 to 0.98 & $<0.001$ & 73 \\
\hline Mortality & Current abstainers & 9 & 853 & 46450 & 0.85 & 0.67 to 1.08 & $<0.001$ & 74 \\
\hline Morbidity & Current abstainers & 2 & 193 & 884 & 0.78 & 0.21 to 2.90 & 0.006 & 87 \\
\hline \multicolumn{9}{|c|}{ Patients with AUD (clinical samples) } \\
\hline Mortality & General population & 9 & 761 & 18758 & 1.62 & 1.34 to 1.95 & $<0.001$ & 81 \\
\hline
\end{tabular}




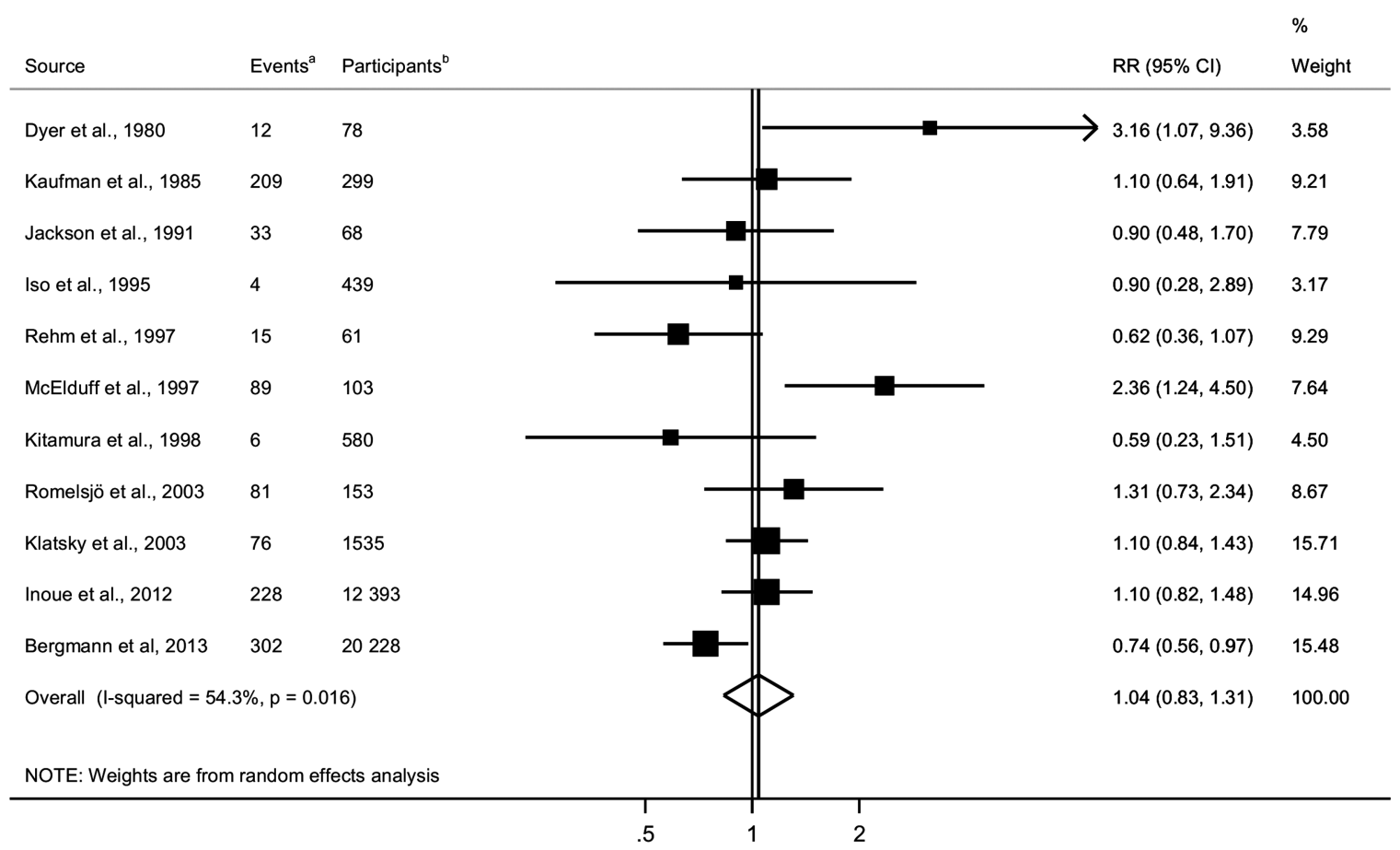

${ }^{a}$ Number of IHD events (fatal and non-fatal) among chronic heavy drinkers

${ }^{\mathrm{b}}$ Number of participants with chronic heavy alcohol consumption

Figure 1 Forest plot of the association between ischaemic heart disease incidence and chronic heavy drinking in population samples in comparison to lifetime abstainers in men, 1980-2012.

heavy drinkers, three with lifetime abstainers $(\mathrm{RR}=1.13$, $95 \%$ CI 0.78 to 1.63 ) and two with current abstainers as the reference group ( $\mathrm{RR}=0.78,95 \%$ CI 0.21 to 2.90$)$. There were not enough studies to further investigate IHD morbidity. Among patients with AUD the risk of IHD mortality in comparison to the general population was substantially elevated with $\mathrm{RR}=1.62(95 \%$ CI 1.34 to 1.95) among men (figure 3).

There were only 114 IHD events reported in women, of which 75 were among patients with AUD. The pooled RR for IHD mortality among patients with AUD ( $n=5$ studies) was 2.09 (95\% CI 1.28 to 3.41, figure 4).

\section{Publication bias and sensitivity analyses}

Tests for publication bias or meta-regressions were not conducted in most analyses because of the small number of studies available for each analysis. Two analyses with enough studies (IHD incidence risk in chronic heavy drinkers compared to lifetime and current abstainers in men) did not reveal evidence for such bias ( $p=0.46$, and 0.35 , respectively, see online supplementary figures S3 and S4 for funnel plots). Omitting studies one by one and re-estimating the pooled risks did not change conclusions in any of the analyses. Using meta-regression, study design (case-control vs cohort studies) did not reveal a significant association for analyses on IHD incidence in chronic heavy drinkers compared to lifetime abstainers $(\mathrm{p}=0.22)$.
The pooled risk for IHD incidence among chronic heavy drinkers in population samples with lifetime abstainers as the reference group that adjusted for age and smoking status $(\mathrm{n}=8$ studies $)$ was virtually unchanged with $\mathrm{RR}=1.04$ (95\% CI 0.82 to 1.33 ). The risk for IHD incidence among chronic heavy drinkers in population samples, which, in addition to age and smoking status, also adjusted for either physical activity, body mass index or at least one socioeconomic indicator was $\mathrm{RR}=0.97 \quad(95 \%$ CI 0.79 to $1.18, \mathrm{n}=6$ studies $)$. A similar sensitivity analysis with current abstainers as the reference group revealed a pooled $\mathrm{RR}=0.75$ (95\% CI 0.62 to 0.91 ) and $\mathrm{RR}=0.72$ (95\% CI 0.58 to 0.89 ). In contrast, only one study among patients with AUD adjusted for risk factors other than age, race, calendar year or follow-up length. Therefore, such analyses were not conducted among clinical samples.

\section{DISCUSSION}

Our analysis showed that when the comparison group was lifetime abstainers in population studies, there was no indication for a protective association from chronic heavy drinking, contrary to our analysis using current abstainers as the reference and results from another meta-analysis, which reported a universal protective association between alcohol consumption and IHD. ${ }^{4}$ However, when current abstainers were the reference 


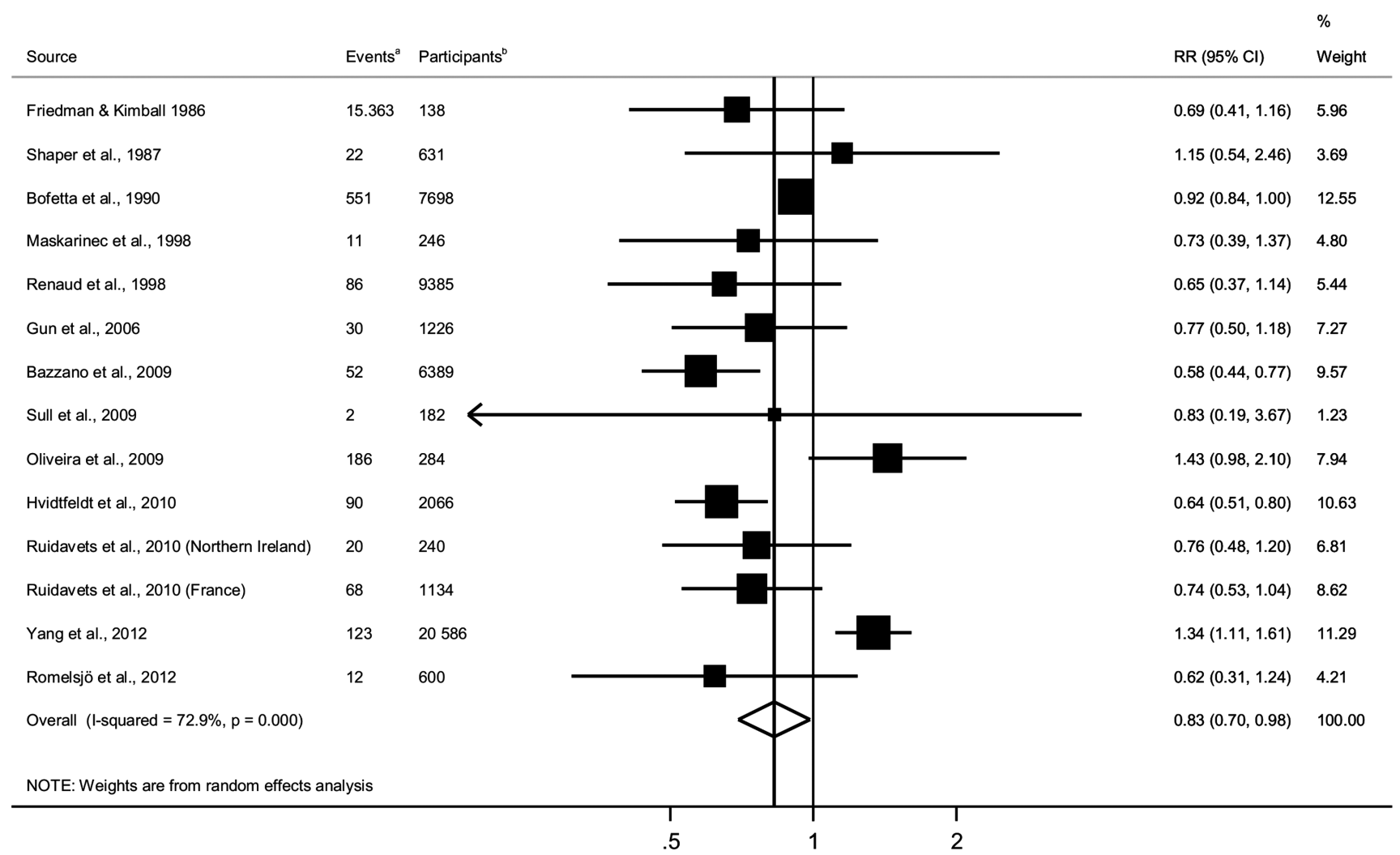

${ }^{a}$ Number of IHD events (fatal and non-fatal) among chronic heavy drinkers

${ }^{\mathrm{b}}$ Number of participants with chronic heavy alcohol consumption

Figure 2 Forest plot of the association between ischaemic heart disease incidence and chronic heavy drinking in population samples in comparison to current abstainers in men, 1986-2012.

group, results indicated a 'protective' association, comparable in magnitude to that found in studies of moderate overall alcohol intake. ${ }^{2}{ }^{4}$ Ronksley $e t a l,{ }^{4}$ similar to Roerecke et $a l^{10}{ }^{10}$ reported that former drinkers had an elevated IHD risk compared to lifetime abstainers. Thus, the difference in IHD risk found in the current meta-analysis is consistent with the fact that the inclusion of former drinkers in the reference group is responsible for the systematic bias in effect estimates when current abstainers are the reference group and leads to erroneous conclusions. Unfortunately, at this point, the majority of studies used current abstainers as the reference group, partly because large studies have limited space for each risk factor and assessing former drinking status requires more questionnaire space and interview time than assessing current abstention. It should be good epidemiological practice to include items that are able to differentiate between former and current drinking status in any epidemiological study on IHD risk.

The difference regarding the reference group was also evident within primary studies, ${ }^{34}$ where the risk was below $R R=1$ when current abstainers were the reference group, and above 1 when lifetime abstainers were the reference group. As most of the studies reported on mortality and only few studies examined IHD morbidity in comparison to lifetime abstainers, we were unable to draw firm conclusions about the relationship between chronic heavy alcohol consumption and IHD morbidity. More systematic research is needed in this area.

With regard to population studies, adjustment for confounders was not optimal in many studies; however subgroup analyses of studies with good adjustment did not change our conclusions (no beneficial association between chronic heavy alcohol consumption and IHD risk). In contrast to chronic heavy drinkers from population samples, clinical samples of patients with AUD showed a clear detrimental association with a $62 \%$ higher risk of heart disease mortality compared to the general population among men, and a twofold higher risk among women. However, there was an apparent lack of adjustment other than age and sex, and lack of recent studies investigating IHD among patients with AUD with only two studies with a baseline assessment after 1990. In particular, the complete lack of adjustment for smoking in clinical samples shows that better quality evidence is needed to confirm the role of alcohol consumption on IHD risk in patients with AUD. As the control group was the general population for clinical samples, the risk for 


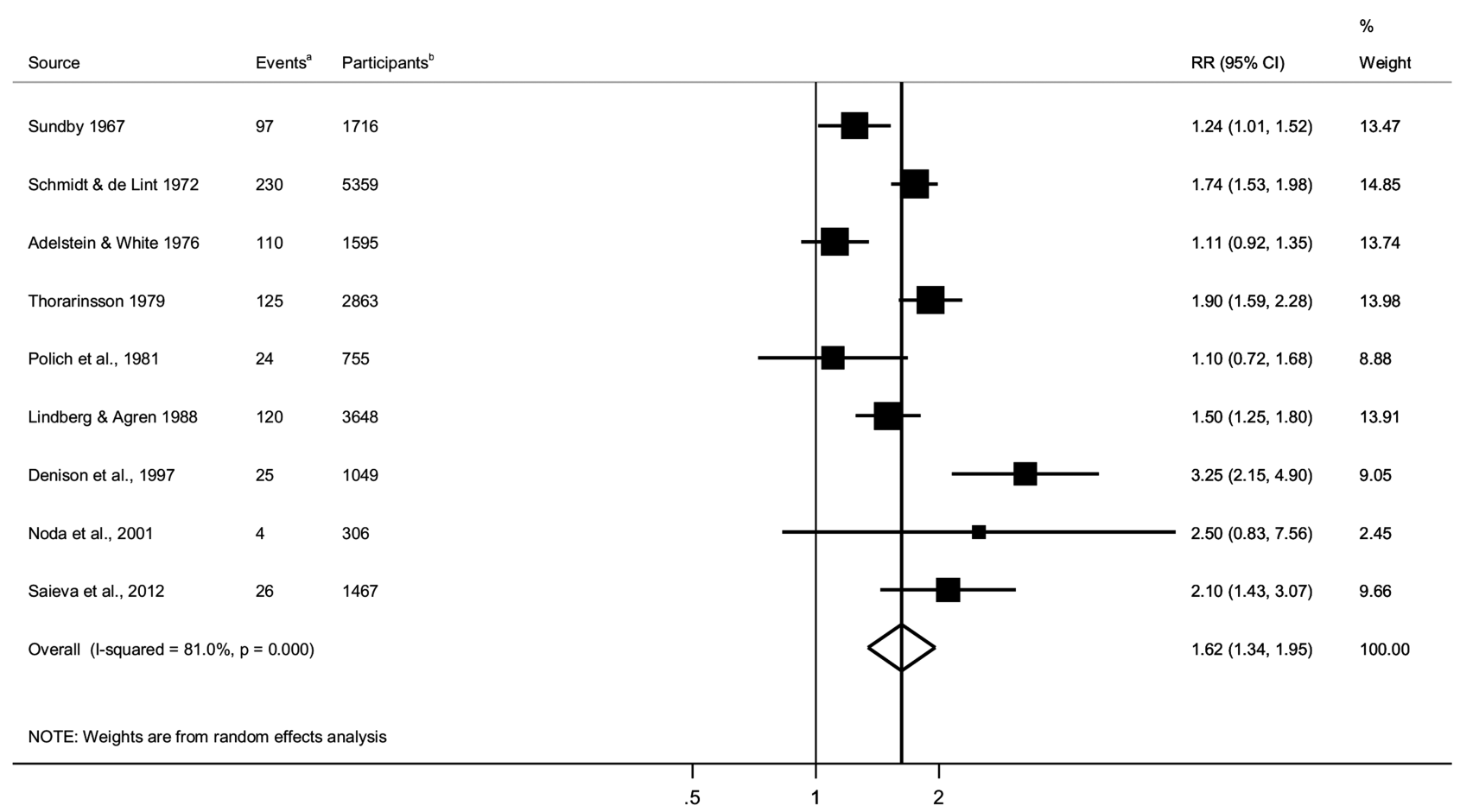

${ }^{a}$ Number of IHD deaths

${ }^{\mathrm{b}}$ Number of patients with alcohol use disorder

Figure 3 Forest plot of the association between ischaemic heart disease mortality and alcohol use disorder in clinical samples in comparison to the general population in men, 1967-2012.

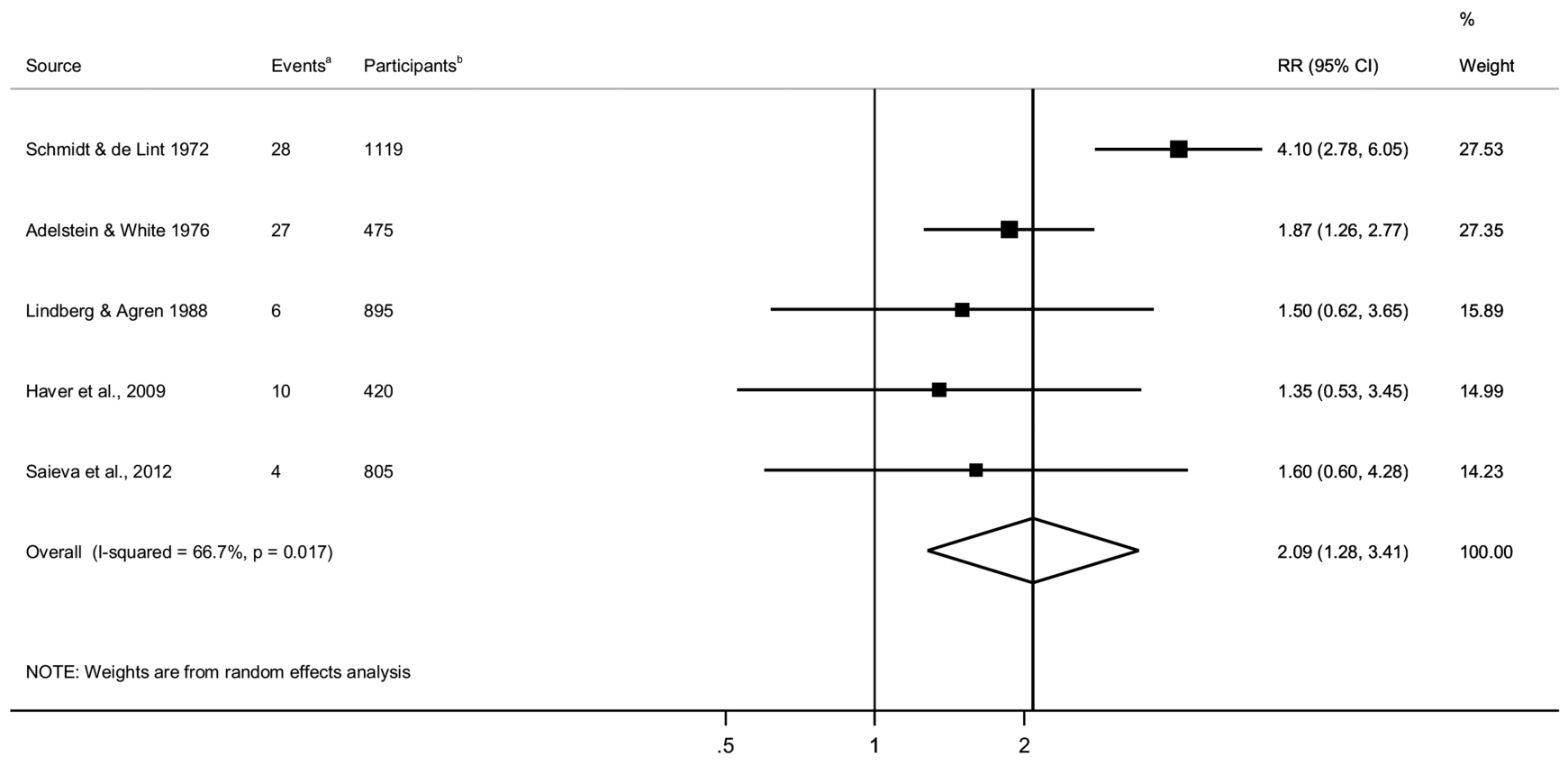

${ }^{\text {a }}$ Number of IHD deaths

${ }^{\mathrm{b}}$ Number of patients with alcohol use disorder

Figure 4 Forest plot of the association between ischaemic heart disease mortality and alcohol use disorder in clinical samples in comparison to the general population in women, 1967-2012. 
IHD should be expected to be somewhat higher compared to population sample results (when the reference was lifetime abstainers) if one assumes a protective effect of moderate alcohol consumption. A recent meta-analysis on average alcohol consumption ${ }^{2}$ has shown strong evidence from observational studies that the risk of IHD among moderate drinkers is reduced in comparison to lifetime abstainers.

Among those reporting the strongest elevated risk for IHD are studies from Russia. ${ }^{36}{ }^{37}$ However, because there are very few abstainers, the reference group is typically not abstainers, but people with low average alcohol intake. A relatively frequent consumption pattern in Russia is episodic heavy to very heavy consumption with sometimes prolonged binges ('zapoi', an episode of continuous drunkenness lasting two or more days in combination with withdrawal from normal social life). This drinking pattern is so extreme that it is heavy with regard to both average and episodic consumption. ${ }^{38} 39$ Nevertheless, the risk among heavy alcohol drinkers in comparison to low level drinkers ${ }^{36} 37$ was substantial, which points to no beneficial effects from heavy alcohol intake and is in support of our findings.

\section{Limitations}

The analysis was limited to English-language, German-language and Spanish-language studies, leaving the possibility of unidentified studies. Furthermore, as is the case for all meta-analyses, our analysis was subject to bias and uncontrolled confounding as they were inherent in the primary studies. $\mathrm{I}^{2}$ values were moderate to high in most analyses. Although adjustment for major IHD risk factors was investigated in population studies and did not result in a change of conclusions, adjustment for risk factors other than age and smoking was not optimal in most studies, and uncontrolled confounding may have contributed to any observed between-study heterogeneity. Uncontrolled confounding might have been most problematic in the clinical samples used in our study, which did not control for smoking or any other IHD risk factor other than sex and age. The choice of random-effect models (although giving more weight to smaller studies) was justified by the amount of heterogeneity detected and because epidemiological studies generally cannot control unobserved confounding in the same way a randomisation process can. However, conclusions were not affected by this choice. Nevertheless, all studies were observational and thus causality cannot be established. Ill-definition of cardiovascular deaths and substantial variation across countries in terms of the quality of classifications of IHD deaths may pose an additional problem. ${ }^{40}$ In a Swedish sample included in our analysis, Denison ${ }^{41}$ examined information from death certificates and independent evaluations from hospital and clinical autopsy reports, police reports, forensic autopsy reports and toxicology reports. They found only a slightly higher mortality risk for cardiovascular diseases compared to death certificate information. As we focused on high-quality epidemiological studies, we excluded self-reported IHD morbidity and other forms of heart disease not defined by ICD-10: I20-I25, and thus cannot generalise beyond the populations and outcomes in our study.

Study quality was substantially lower in clinical samples because of lack of adjustment for potential confounding. Only one study ${ }^{15}$ reported risk estimates adjusted for more than just age, one other study also adjusted for length of follow-up. ${ }^{41}$ Thus, confounding or effect modification from factors other than age and sex could not be examined. It seems likely that, given the close correlation of smoking with alcohol consumption in general and at high levels of alcohol consumption in particular, smoking had some undetected influence on IHD mortality in clinical samples because of a clearly established monotonous detrimental relationship of smoking with heart disease. However, there is surprisingly little research on this topic and it remains to be seen whether smoking explains the elevated risk for IHD seen in patients with AUD. Few studies have examined IHD risk stratified by alcohol and smoking in population studies, ${ }^{34} 4243$ but no clear picture emerged. In particular, there are no studies investigating potential joint effects from smoking and chronic heavy alcohol consumption on IHD risk with lifetime abstainers as the reference group. If alcohol consumption in patients with AUD is the determining factor for an increased risk of IHD, evidence from AUD treatment outcome studies could provide further pieces of evidence for a potential causal effect. Many studies among patients with AUD showed that a reduction from chronic heavy drinking to moderate or low levels, including but not limited to abstention, can substantially reduce all-cause mortality. ${ }^{44}$ However, there seems to be no investigations regarding whether or not IHD as a cause of death played a substantial role in this reduction of all-cause mortality.

\section{Experimental evidence for chronic heavy drinking and heart disease risk}

What is the underlying experimental evidence base? Long-term randomised studies on IHD mortality or morbidity are unavailable. Although regular low to moderate alcohol intake has been found to have beneficial, dosedependent effects on biomarkers for IHD in short-term experimental studies, mainly by increasing high-density lipoprotein (HDL) levels, inhibiting platelet activation, reducing fibrinogen levels and producing antiinflammatory effects, ${ }^{6}$ chronic heavy drinking has been found to be related to detrimental effects on the heart, with adverse effects mainly on blood pressure, fibrinolytic factors and ventricular arrhythmia after cessation of heavy drinking, as well as in participants with existing ischaemic disease through silent myocardial ischaemia and angina. ${ }^{45}$ Chronic alcohol intake in particular is associated with physiological changes of the heart, including prolonged QT intervals and electrolyte abnormalities. ${ }^{46}$ There are some short-term experimental 
studies specifically on regular heavy drinking. During heavy alcohol intake, trygliceride levels were elevated in most studies, ${ }^{47-50}$ with transient positive effects on trygliceride in one study $y^{51}$ and HDL cholesterol was elevated in all studies. ${ }^{47-52}$ In fact, the highest HDL levels are observed in people with AUD. ${ }^{53}$ It seems that despite a beneficial effect on HDL cholesterol even in chronic heavy alcohol consumers, other effects of chronic heavy alcohol consumption on heart disease risk might negate those beneficial effects, ${ }^{54}{ }^{55}$ resulting in an overall neutral or detrimental association found in our analysis. Further work on distinct biochemical pathways and differentiation of heart disease outcomes should be a priority in alcohol-heart research.

\section{Conclusions}

As the evidence base is scarce in women, we restrict our conclusions to men only. Our findings, in combination with previous investigations, ${ }^{2}{ }^{20}$ lead to three main conclusions: First, there is no systematic evidence of a protective association between IHD and chronic heavy drinking. Second, the findings show that the reference category is crucial in determining IHD risk from any type of alcohol consumption. Public perception of a universal cardioprotective association, however, might overshadow these important parts of the alcohol-heart relationship, as it can be used as an excuse for heavier drinking. Third, a detrimental association of alcohol consumption on IHD is evident only for patients with AUD, the highest end of the spectrum of alcohol consumption. It should be stressed that there is a clear detrimental effect of any heavy drinking episodes on injuries and through overall intake on many cancers. ${ }^{56} 57$ Thus heavy drinking in all forms should be discouraged.

Contributors All authors have contributed to the writing of this review article and are responsible for the overall content.

\section{Competing interests None.}

Funding The research leading to these results or outcomes has received funding from the European Community's Seventh Framework Programme (FP7/2007-2013), under Grant Agreement $n^{\circ} 266813$ - Addictions and Lifestyle in Contemporary Europe - Reframing Addictions Project (ALICE RAP - www.alicerap.eu). Participant organisations in ALICE RAP can be seen at http://www.alicerap.eu/about-alice-rap/partner-institutions.html. The views expressed here reflect only the author's and the European Union is not liable for any use that may be made of the information contained therein.

Provenance and peer review Not commissioned; externally peer reviewed.

Data sharing statement No additional data are available.

Open Access This is an Open Access article distributed in accordance with the Creative Commons Attribution Non Commercial (CC BY-NC 3.0) license, which permits others to distribute, remix, adapt, build upon this work noncommercially, and license their derivative works on different terms, provided the original work is properly cited and the use is non-commercial. See: http:// creativecommons.org/licenses/by-nc/3.0/

\section{REFERENCES}

1. Jackson R, Broad J, Connor J, et al. Alcohol and ischaemic heart disease: probably no free lunch. Lancet 2005;366:1911-12.
2. Roerecke M, Rehm J. The cardioprotective association of average alcohol consumption and ischaemic heart disease: a systematic review and meta-analysis. Addiction 2012;107:1246-60.

3. Naimi TS, Brown DW, Brewer RD, et al. Cardiovascular risk factors and confounders among nondrinking and moderate-drinking US adults. Am J Prev Med 2005;28:369-73.

4. Ronksley PE, Brien SE, Turner BJ, et al. Association of alcohol consumption with selected cardiovascular disease outcomes:a systematic review and meta-analysis. BMJ 2011;342:d671.

5. Corrao G, Rubbiati L, Bagnardi V, et al. Alcohol and coronary heart disease: a meta-analysis. Addiction 2000;95:1505-23.

6. Brien SE, Ronksley PE, Turner BJ, et al. Effect of alcohol consumption on biological markers associated with risk of coronary heart disease: systematic review and meta-analysis of interventional studies. BMJ 2011;342:d636.

7. Sattelmair J, Pertman J, Ding EL, et al. Dose response between physical activity and risk of coronary heart disease: a meta-analysis. Circulation 2011;124:789-95.

8. Roger VL, Go AS, Lloyd-Jones DM, et al. Heart disease and stroke statistics-2012 update: a report from the American Heart Association. Circulation 2012;125:e2-220.

9. Maclure M. Demonstration of deductive metaanalysis-ethanol intake and risk of myocardial infarction. Epidemiol Rev 1993;15:328-51.

10. Roerecke M, Rehm J. Ischemic heart disease mortality and morbidity in former drinkers: a meta-analysis. Am J Epidemiol 2011;73:245-58.

11. Rothman KJ, Greenland S, Lash TL. Modern epidemiology. 3rd edn Philadelphia, PA: Lippincott Williams \& Wilkins, 2008.

12. Mukamal K, Conigrave K, Mittleman M, et al. Roles of drinking pattern and type of alcohol consumed in coronary heart disease in men. N Engl J Med 2003;348:109-18.

13. World Health Organization. Global status report on alcohol and health. Geneva, Switzerland: World Health Organization, 2011.

14. Rehm J, Anderson P, Gual A, et al. The tangible common denominator of substance use disorders: a reply to commentaries to Rehm et al (2013). Alcohol Alcohol 2014;49:118-22.

15. Haver B, Gjestad R, Lindberg S. Mortality risk up to 25 years after initiation of treatment among 420 Swedish women with alcohol addiction. Addiction 2009;104:413-19.

16. Rivas I, Sanvisens A, Bolao F, et al. Impact of medical comorbidity and risk of death in 680 patients with alcohol use disorders. Alcohol Clin Exp Res 2012;37:E221-7.

17. Noda T, Imamichi H, Tanaka H. Cause-specific mortality risk among male alcoholics residing in the Osaka metropolitan area. Psychiatry Clin Neurosci 2001;55:465-72.

18. Saieva C, Bardazzi G, Masala G, et al. General and cancer mortality in a large cohort of Italian alcoholics. Alcohol Clin Exp Res 2012;36:342-50.

19. Stroup DF, Berlin JA, Morton SC, et al. Meta-analysis of observational studies in epidemiology: a proposal for reporting JAMA 2000;283:2008-12.

20. Roerecke M, Rehm J. Irregular heavy drinking occasions and risk of ischemic heart disease: a systematic review and meta-analysis. Am $J$ Epidemiol 2010;171:633-44.

21. Roerecke M, Rehm J. Alcohol use disorders and mortality-a systematic review and meta-analysis. Addiction 2013;108:1562-78.

22. Gmel G, Kuntsche E, Rehm J. Risky single occasion drinking: bingeing is not bingeing. Addiction 2011;106:1037-45.

23. Tunstall-Pedoe $\mathrm{H}$, Kuulasmaa $\mathrm{K}$, Amouyel $\mathrm{P}$, et al. Myocardial infarction and coronary deaths in the World Health Organization MONICA Project. Registration procedures, event rates, and case-fatality rates in 38 populations from 21 countries in four continents. Circulation 1994;90:583-612.

24. Rose GA, Blackburn H. Cardiovascular survey methods. Geneva, Switzerland, 1982

25. Moher D, Pham B, Jones A, et al. Does quality of reports of randomised trials affect estimates of intervention efficacy reported in meta-analyses? Lancet 1998;352:609-13.

26. Chalmers TC, Smith HJ, Blackburn B, et al. A method for assessing the quality of a randomized control trial. Control Clin Trials 1981:2:31-49.

27. Detsky AS, Naylor CD, O'Rourke K, et al. Incorporating variations in the quality of individual randomized trials into meta-analysis. $J$ Clin Epidemiol 1992;45:255-65.

28. Greenland S, O'Rourke K. On the bias produced by quality scores in meta-analysis, and a hierarchical view of proposed solutions. Biostatistics 2001;2:463-71.

29. Herbison P, Hay-Smith J, Gillespie WJ. Adjustment of metaanalyses on the basis of quality scores should be abandoned. J Clin Epidemiol 2006;59:1249-56. 
30. Hamling J, Lee $\mathrm{P}$, Weitkunat R, et al. Facilitating meta-analyses by deriving relative effect and precision estimates for alternative comparisons from a set of estimates presented by exposure level or disease category. Stat Med 2008;27:954-70.

31. DerSimonian R, Laird N. Meta-analysis in clinical trials. Control Clin Trials 1986;7:177-88.

32. Higgins JP, Thompson SG. Quantifying heterogeneity in a metaanalysis. Stat Med 2002;21:1539-58.

33. Egger M, Smith GD, Schneider M, et al. Bias in meta-analysis detected by a simple, graphical test. BMJ 1997;315:629-34.

34. Inoue M, Nagata C, Tsuji I, et al. Impact of alcohol intake on total mortality and mortality from major causes in Japan: a pooled analysis of six large-scale cohort studies. J Epidemiol Community Health 2012;66:448-56.

35. Hvidtfeldt UA, Tostrup JS, Jakobsen MU, et al. Alcohol intake and risk of coronary heart disease in younger, middle-aged, and older adults. Circulation 2010;121:1589-97.

36. Zaridze D, Lewington S, Boroda A, et al. Alcohol and mortality in Russia: prospective observational study of 151,000 adults. Lancet 2014;383:1465-73.

37. Zaridze D, Brennan P, Boreham J, et al. Alcohol and cause-specific mortality in Russia: a retrospective case-control study of 48,557 adult deaths. Lancet 2009;373:2201-14.

38. Tomkins S, Saburova L, Kiryanov N, et al. Prevalence and socioeconomic distribution of hazardous patterns of alcohol drinking: study of alcohol consumption in men aged 25-54 years in Izhevsk, Russia. Addiction 2007;102:544-53.

39. Malyutina S, Bobak M, Kurilovitch S, et al. Relation between heavy and binge drinking and all-cause and cardiovascular mortality in Novosibirski, Russia: a prospective cohort study. Lancet 2002;360:1448-54

40. Lozano R, Murray CJL, Lopez AD, et al. Miscoding and misclassification of ischaemic heart disease mortality. Global program on evidence for health policy discussion paper 12. Geneva, Switzerland, 2001.

41. Denison $\mathrm{H}$, Berkowicz $\mathrm{A}$, Oden $\mathrm{A}$, et al. The significance of coronary death for the excess mortality in alcohol-dependent men. Alcohol Alcohol 1997;32:517-26.

42. Hart CL, Davey Smith G, Gruer L, et al. The combined effect of smoking tobacco and drinking alcohol on cause-specific mortality: a 30 year cohort study. BMC Public Health 2010;10:789.

43. Ebbert JO, Janney CA, Sellers TA, et al. The association of alcoho consumption with coronary heart disease mortality and cancer incidence varies by smoking history. J Gen Intern Med 2005;20:14-20.
44. Rehm J, Roerecke M. Reduction of drinking in problem drinkers and all-cause mortality. Alcohol Alcohol 2013;48:509-13.

45. Puddey IB, Rakic V, Dimmitt SB, et al. Influence of pattern of drinking on cardiovascular disease and cardiovascular risk factorsa review. Addiction 1999;94:649-63.

46. George A, Figueredo VM. Alcohol and arrhythmias: a comprehensive review. J Cardiovasc Med 2010;11:221-8.

47. Contaldo F, D'Arrigo E, Carandente V, et al. Short-term effects of moderate alcohol consumption on lipid metabolism and energy balance in normal men. Metabolism 1989;38:166-71.

48. Malmendier CL, Delcroix C. Effect of alcohol intake on high and low density lipoprotein metabolism in healthy volunteers. Clin Chim Acta 1985;152:281-8.

49. Crouse JR, Grundy SM. Effects of alcohol on plasma lipoproteins and cholesterol and triglyceride metabolism in man. $J$ Lipid Res 1984;25:486-96.

50. Belfrage P, Berg B, Cronholm T, et al. Prolonged administration of ethanol to young, healthy volunteers: effects on biochemical, morphological and neurophysiological parameters. Acta Med Scand 1973;552:1-44.

51. Belfrage $\mathrm{P}$, Berg B, Hagerstrand I, et al. Alterations of lipid metabolism in healthy volunteers during long-term ethanol intake. Eur J Clin Invest 1977;7:127-31.

52. Välimäki M, Laitinen K, Ylikahri R, et al. The effect of moderate alcohol intake on serum apolipoprotein A-I-containing lipoproteins and lipoprotein (a). Metabolism 1991;40:1168-72.

53. Seppä K, Sillanaukee P, Pitkäjärvi T, et al. Moderate and heavy alcohol consumption have no favorable effect on lipid values. Arch Intern Med 1992;152:297-300.

54. Peasey A, Bobak M, Malyutina S. Do lipids contribute to the lack of cardio-protective effect of binge drinking: alcohol consumption and lipids in three eastern European countries. Alcohol Alcohol 2005;40:431-5

55. Foerster M, Marques-Vidal P, Gmel G, et al. Alcohol drinking and cardiovascular risk in a population with high mean alcohol consumption. Am J Cardiol 2009;103:361-8.

56. Whiteford HA, Degenhardt L, Rehm J, et al. Global burden of disease attributable to mental and substance use disorders: findings from the Global Burden of Disease Study 2010. Lancet 2013;382:1575-86.

57. Rehm J, Baliunas D, Borges GLG, et al. The relation between different dimensions of alcohol consumption and burden of disease -an overview. Addiction 2010;105:817-43. 